HStud 23 (2009)1, 11-28 DOI: 10.1556/HStud.23.2009.1.2

\title{
MEDIZIN UND MEDIZINER IM ZEITALTER VON FERENC KAZINCZY
}

\author{
LILLA KRÁSZ \\ Eötvös-Loránd-Universität, Budapest \\ Ungarn
}

\begin{abstract}
Das Zeitalter von Ferenc Kazinczy war eine entscheidende Phase in der spektakulären Durchsetzung ärztlicher Deutungsmacht. Die gelehrte Medizin der promovierten Ärzte wurde von England über Frankreich bis hin zum Habsburgerreich, so auch in Ungarn, von immer größeren Bevölkerungsgruppen nachgefragt. Die im vorliegenden Aufsatz behandelten sechs Ärzte (József Szentgyörgyi, József Viczay, Sámuel Nagy, József Cseh-Szombati, Mihály Kováts und János Dercsényi) repräsentieren das anspruchsvolle Interesse an kulturellen Erfahrungen, das enzyklopädische Wissen, den in der Gesellschaft eingenommenen Status und den gesellschaftlichen Rang, somit all das, was für eine kleinere Elitegruppe der akademischen Ärzteschaft im Zeitalter der Aufklärung kennzeichnend war.
\end{abstract}

Schlüsselwörter: Aufklärung, Ferenc Kazinczy, Medizin, medizinische Aufklärungsliteratur, Gesundheitsmarkt, akademische Ärzteschaft, vitalistische Heilungskonzepte / Vitalismus, medizinisches Laienwissen

Das Zeitalter von Ferenc Kazinczy war eine entscheidende Phase in der spektakulären Durchsetzung ärztlicher Deutungsmacht. Die gelehrte Medizin der promovierten Ärzte wurde von immer größeren Bevölkerungsgruppen angenommen. ${ }^{1}$ Von England über Frankreich bis hin zum Habsburgerreich vervielfachte sich die Zahl der akademischen Ärzte. Ihre Erklärungsmodelle und Praktiken fanden weithin Anerkennung, aber gleichzeitig mussten sie den Wert und die Gültigkeit ihrer besonderen Expertise wiederholt unter Beweis stellen. Sie mussten einerseits eine anhaltend skeptische Öffentlichkeit, andererseits eine Vielzahl von konkurrierenden Heilkundigen auf dem Markt der Gesundheitsversorgung bekämpfen. ${ }^{2}$

Die Quellen verschiedener Gattung - amtliche Akten, ärztliche Sanitätsberichte, kaiserliche und königliche Erlässe, private Korrespondenzen/Patientenbriefe verraten gleichzeitig viel über die Offenheit der verschiedenen gesellschaftlichen Schichten gegenüber den modernen Sanitätsrichtlinien, Krankheitskonzepten und Therapieformen. Diese Epoche war im Sanitätswesen vom Räsonieren zwischen den akademischen Ärzten und den empirischen Heilkundigen, den fachgerecht in 
Punkte gefassten Normativen und den fachlich nur schwer oder gar nicht erfassbaren Heilmethoden, also von einem fruchtbaren Dialog, einem Zusammenwirken zwischen scientia medica und ars medica geprägt. $^{3}$

\section{Vielfältige Heilkundige - vielfältige Heilungskonzepte}

Um die Tätigkeit der um die 18-19. Jahrhundertwende wirkenden Ärzte sowie ihre steigende gesellschaftliche Position nachzuvollziehen, werfen wir einen Blick auf den vielfältigen und stark besetzten Gesundheitsmarkt (Tabelle 1.) dem die Ärzte ebenso wie die anderen Akteure angehörten. Die Arbeitsteilung, die seit dem Mittelalter Europaweit die gelehrten Ärzte von den anderen Schichten der Heilkundigen unterschied, blieb in der von uns behandelten Epoche bestehen.

Neben den offiziell tätigen studierten Ärzten, die diagnostizierten, Behandlun-

Tabelle 1.

Protagonisten des frühneuzeitlichen Gesundheitsmarktes in Ungarn

\begin{tabular}{ll}
\hline UNGEBILDETE/INOFFIZIELLE & AKADEMISCHE HEILKUNDIGEN \\
HEILKUNDIGEN & $\begin{array}{l}\text { diplomierte Ärzte (Doctor medicinae) } \\
\text { diplomierte Chirurgen (Doctor chirurgiae) }\end{array}$ \\
\hline \begin{tabular}{l} 
WANDERSPEZIALISTEN \\
Steinmetzer (Lithotomus) \\
Okulisten \\
$\begin{array}{l}\text { Zahnzieher (Dentrifrangibuli) } \\
\text { wandernde Arzneihändler, Olitätenhändler } \\
\text { (olearii), Materialisten, Drogisten }\end{array}$ \\
\hline ANSÄSSIGE HEILKUNDIGEN
\end{tabular} \\
$\begin{array}{l}\text { Kräuterfrauen/-männer } \\
\text { Bauerhebammen }\end{array}$ \\
$\begin{array}{l}\text { Kurpfuscher, Quacksalber } \\
\text { Henker }\end{array}$ & GEBILDETE/OFFIZIELLE HEILKUNDIGEN \\
\hline
\end{tabular}

gen verschrieben und Rezepte ausstellten, finden wir die Operateure, die manuell die blutigen Eingriffe vornahmen, also die geprüften Chirurgen und die Barbiere. Diese schlossen sich mehrheitlich in Zünften zusammen, vom letzten Drittel des 18. Jahrhunderts an arbeiteten sie meistens unabhängig. Ebenfalls in Zünften organisiert waren die auf die Krankenversorgung der anspruchsvollen Mittelschicht spezialisierten Bader, die das Schröpfen und die Balneotherapien vornahmen. $\mathrm{Zu}$ den offiziellen Heilkundigen gehörten auch die geprüften Apotheker und diplomierten Hebammen. Diese Schichten der Heilkundigen - von den akademischen Ärzten bis zu den diplomierten Hebammen - ließen sich beinahe ausschließlich 
in den freien königlichen Städten oder den größeren Marktflecken nieder. Ihre Dienste konnten von Familien der Aristokratie, des mittleren Adels und vom städtischen Mittelstand (Intellektuellen, wohlhabenden Handwerkern und Händlern) in Anspruch genommen werden. Der Großteil der Bevölkerung Ungarns, der gemeine Mann in den Marktflecken, Dörfern oder auf Gütern wandte sich mit seinen Krankheiten an einen Spezialisten im weiten Kreis der inoffiziellen, im ganzen Land tätigen Wanderpfuscher oder lokalen Heilkundigen. ${ }^{4}$ Für die gelehrten Fachmänner der Zeit, die akademischen Ärzte, die Chirurgen und die Apotheker, sowie die diplomierten Hebammen bedeuteten die zahlreichen inoffiziellen Spezialisten eine große Herausforderung und nicht zuletzt einen starken Wettbewerb. Eine wichtige, auch zahlenmäßig bedeutende Schicht der nicht ausgebildeten, inoffiziellen, überall auftauchenden Heilkundigen waren die Wanderpfuscher. Sie zogen von Stadt zu Stadt und erhöhten durch ihre Anwesenheit die bunte Vielfalt der zahlreich besuchten Märkte. Die Bruch- und Steinschneider (Herniotomus, Lithotomus), die Zahnzieher (Dentrifrangibuli) und die Augenoperationen durchführenden Okulisten waren die gefragtesten Spezialisten auf den Jahres- oder Wochenmärkten. Unter den Wanderspezialisten hoch geschätzt und überall bekannt waren die mehrheitlich aus Oberungarn stammenden slowakischen Olitätenhändler (Olearii) und andere fahrende Arzneimittelhändler. Sie boten teils selbst hergestellte, teils angekaufte ätherische Öle und Destillate feil. Als ihre exklusivste Ware galt der vom Pinus Cembra destillierte Balsam der Karpaten (Balsamum Carpathicum). Sie handelten nicht nur mit ihren Arzneien, deren Zusammensetzung äußerst fragwürdig war, sondern wandten diese zur Heilung von Krankheiten an, die keine Eingriffe benötigten. ${ }^{5}$ Ein ebenso vielfältiges Bild bieten die Heilkundigen mit festem Wohnsitz - die männlichen und weiblichen medizinischen Spezialisten -, die auf dem Gesundheitsmarkt des 18. Jahrhunderts eine gleichfalls wichtige Rolle spielten. Sie eigneten sich ihr Wissen beinahe seit ihrer Geburt an, lernten die Griffe ihres Handwerks von ihren Verwandten, Nachbarn oder von bereits erfolgreichen und anerkannten Heilkundigen in der alltäglichen Praxis. Erfahrung, geschickte Hände und Bekanntheit in der jeweiligen Gemeinde sowie der überlieferte gute Ruf konnte ihnen Anerkennung und nicht zuletzt das Einkommen sichern. Die Palette war auch hier breit: Kräuterfrauen, weise Frauen, Knochenbrecher, narkotisierende und chirurgische Eingriffe vornehmende Henker, Bauernhebammen - um nur die wichtigsten Tätigkeitsgruppen zu nennen. Diese Heilkundigen waren wie die anderen Mitglieder ihrer Gemeinde Bäuerinnen, Witwen städtischer Barbiere oder Chirurgen, dörfliche Geistliche, Priester oder gar Mönche, Scharfrichter, Hufschmiede, die die ars medica, das Handwerk des Heilens nebenberuflich ausübten - manche aus finanzieller Notwendigkeit, andere aus Menschenliebe oder aus bloßem Interesse an der Heilung. 
Ein grundsätzlicher Unterschied zwischen dem Fachwissen und den Behandlungsmethoden der akademischen und nichtakademischen Heilkundigen oder medizinischen Laien lässt sich in der alltäglichen Praxis rückblickend nicht erkennen. Die Grundlage physiologischer und pathologischer Überlegungen war immer noch die hippokratisch-galenische Vier-Säfte-Lehre (Tabelle 2.), nach der die meisten Krankheiten durch überschüssige oder verdorbene Säfte hervorgerufen wurden. Die dementsprechende Behandlung mit Aderlässen, Schröpfen, Abführ- und Brechmitteln, Zugpflastern, schweißtreibenden Arzneien und ähnlich entleerenden Verfahren blieb das gesamte 18. Jahrhundert hindurch auch in der ärztlichen Praxis bestimmend. In der Deutung konkreter Körperfunktionen und Krankheitserscheinungen zeichnete sich die akademische Medizin im Allgemeinen durch große Vielfalt und Widersprüchlichkeit aus. Die ärztlichen Sanitätsberichte aus den 1780er Jahren belegen, dass es kaum eindeutige Standardregeln gab, wie bestimmte Krankheiten verstanden und behandelt werden mussten, ob es um Schwindsucht, Scharbock oder Wassersucht ging.

Tabelle 2.

Vier-Säfte-Lehre (Humoralpathologie)

\begin{tabular}{lllll}
\hline 4 ELEMENTE & Luft & Feuer & Wasser & Erde \\
4 QUALITÄTEN & warm, feucht & warm, trocken & kalt, feucht & kalt, trocken \\
4 SÄFTE (HUMORES) & Blut & gelbe Galle & Schleim & schwarze Galle \\
4 ORGANE & Herz & Galle & Gehirn & Verdauungssystem \\
4 TEMPERAMENTE & sanguinisch & kolerisch & flegmatisch & melankolisch \\
\hline
\end{tabular}

Die akademische Medizin hatte einen aktiven Anteil am Trend zur Empirie, persönlichen Beobachtung und Forschung. Der Raum, wo sich die staatlich legitimierte Medizin neu formierte, allgemein gültige wissenschaftliche Standards und ein neues Verhältnis von Theorie und Praxis eingerichtet wurden, war die Universität. Es waren die medizinischen Fakultäten, die infolge des Aufstiegs der Anatomie und Pathologie zur Basiswissenschaft der Medizin die Empirie regulierten, Aufschreibtechniken wie Fallberichte und statistische Tabellen weiterentwickelten und die klinische Praxis in den Unterricht einführten. Im Allgemeinen erlangte das Experiment durch den Import von Leitkonzepten aus Physik und Chemie eine zunehmende Bedeutung. Die praktische Umsetzung einer auf Anatomie und Pathologie sowie von dem Leidener Professor Hermann Boerhaave (1668-1738) propagierter klinischer Erfahrung basierenden Medizin war ein langwieriger Prozess, dessen Modalitäten mehrere Generationen von Ärzten immer wieder neu aushandeln mussten. ${ }^{6}$

Die medizinischen Gelehrten in Ungarn orientierten sich in erster Linie an den deutschen protestantischen Universitäten - vor allem an den medizinischen $\mathrm{Fa}$ - 
kultäten in Halle, Jena und Göttingen - wo die neuesten sog. vitalistischen Gesundheits- und Krankheitskonzepte (Tabelle 3.) allmählich eine systembildende Bedeutung gewannen. Die besondere Bewertung der Aufregungen aller Art (physikalischer/psychischer) in ihrer Rolle für Gesundheit und Krankheit durchzieht seit der Mitte des 18. Jahrhunderts die gesamte medizinische Literatur. Die philosophische Grundlage der vitalistischen Krankheitskonzepte bildete die Überzeugung, dass die Affekte, Leidenschaften die Widersacher der Vernunft seien, die aber wohl beherrscht werden sollten. Nur mit ausgeglichenem Gemüt kann der Mensch zu wahrer Erkenntnis, zum Vorherrschen der Vernunft, zu seinem Glück und zur wahren Glückseligkeit gelangen. Vernunft und Besonnenheit gelten als

Tabelle 3.

Vitalistische Krankheitskonzepte im 18. Jahrhundert

\begin{tabular}{|c|c|c|c|}
\hline G. E. Stahl & $\begin{array}{l}\text { „, Theoria medica } \\
\text { vera“ (1707) }\end{array}$ & $\begin{array}{l}\text { animistische-vitalistische } \\
\text { Lehre }\end{array}$ & $\begin{array}{l}\text { anregende und } \\
\text { beruhigende Mittel, } \\
\text { Chinarinde, Opium }\end{array}$ \\
\hline Fr. Hoffmann & $\begin{array}{l}\text { „Medicina rationalis } \\
\text { systematica“" } \\
(1718-1740)\end{array}$ & $\begin{array}{l}\text { mechanische und } \\
\text { physiologische } \\
\text { Tonusstörung }\end{array}$ & $\begin{array}{l}\text { Reinigungsmittel, } \\
\text { anregende und } \\
\text { beruhigende Mittel }\end{array}$ \\
\hline H. Boerhaave & $\begin{array}{l}\text { „Aphorismi“" } \\
(1709)\end{array}$ & $\begin{array}{l}\text { vermehrte/verminderte } \\
\text { Herzkreislaufaktion }\end{array}$ & $\begin{array}{l}\text { Abführ- oder Brech- } \\
\text { mittel, anregende und } \\
\text { beruhigende Mittel }\end{array}$ \\
\hline A. von Haller & $\begin{array}{l}\text { „Elementa } \\
\text { physiologiae“ } \\
(1757-1766)\end{array}$ & $\begin{array}{l}\text { Irritabilitäts- und } \\
\text { Sensibilitätslehre }\end{array}$ & $\begin{array}{l}\text { Abführ- oder Brech- } \\
\text { mittel, anregende und } \\
\text { beruhigende Mittel }\end{array}$ \\
\hline J. Brown & $\begin{array}{l}\text { „Elementa } \\
\text { medicinae“ (1780) }\end{array}$ & $\begin{array}{l}\text { Reaktionsfähigkeit auf } \\
\text { äußere und innere Reize }\end{array}$ & $\begin{array}{l}\text { Abführ- oder Brech- } \\
\text { mittel, anregende und } \\
\text { beruhigende Mittel }\end{array}$ \\
\hline Ch. W. Hufeland & $\begin{array}{l}\text { „Makrobiotik“ } \\
\text { (1797) }\end{array}$ & $\begin{array}{l}\text { Verstärkung der } \\
\text { Selbstheilungsfähigkeit } \\
\text { der Organismen }\end{array}$ & $\begin{array}{l}\text { Körperbewegung, } \\
\text { Diätkuren, Abführ- } \\
\text { oder Brechmittel, } \\
\text { anregende und } \\
\text { beruhigende Mittel }\end{array}$ \\
\hline F. A. Mesmer & $\begin{array}{l}\text { „Mémoires sur } \\
\text { magnétisme animal“ } \\
(1779)\end{array}$ & $\begin{array}{l}\text { Mesmerizmus } \\
\text { (,tierischer } \\
\text { Magnetismus“) }\end{array}$ & $\begin{array}{l}\text { suggestive } \\
\text { Magnetisierung }\end{array}$ \\
\hline S. Hahnemann & „Organon“ (1810) & $\begin{array}{l}\text { „Simila similibus } \\
\text { curantur“ }\end{array}$ & $\begin{array}{l}\text { Chinarinde, } \\
\text { Verdünnung }\end{array}$ \\
\hline
\end{tabular}


höchstes Gut des aufgeklärten Menschen, sie verbürgen die Gesundheit. „Vernünftige Gedanken“ und vernünftiges Verhalten alleine können die Welt verbessern. $^{7}$

In der Medizinwissenschaft des 18. Jahrhunderts galt Friedrich Hoffmann (1660-1742) als der große „Systematiker“. Als Universitätsprofessor in Halle entwickelte er ein neues Konzept von Krankheit, überdies ein systematisch aufgebautes, logisch gegliedertes Konzept der gesamten Medizin, welches seine innere Geschlossenheit, Schlüssigkeit und Lehrbarkeit durch die enge Verbindung mit der Natur- und Menschenlehre Descartes' erhielt. Aus zwei Wurzeln, aus ratio und experientia ist nach Hoffmann das Gebäude der Medizin zu entwickeln. Ratio liefert die Grundsätze, die principia, Anatomie, Physiologie, Chemie und die Krankenbeobachtung (historia morborum) liefern die Erfahrungsmaterialien für den Aufbau der Pathologie. ${ }^{8}$ Seine physiologische Hauptlehre bestand darin, dass alle Körper aus tonusbegabten Fasern bestünden, die kontraktil und erschlaffbar seien und die durch einen „Nervenaether", dessen Sitz im Gehirn sei, angeregt würden. Er behandelte mit Diät, beruhigenden oder aber anregenden Mitteln und erfand auch mehrere Arzneien. ${ }^{9}$ Den von Hoffmann geführten „Systematiker“ standen die „Animisten“ gegenüber, deren geistiger Vater Georg Ernst Stahl (1660-1734), Hoffmanns Fakultätsgenosse in Halle war. Seine Schule lehrte, dass die Seele, die Anima, der Mittelpunkt allen Geschehens sei. Der Körper sei nur ihr Werkzeug und alle Krankheiten des Körpers gingen auf Erkrankungen der Seele zurück. Die Arbeiten des Göttinger Polyhistor-Physiologen Albrecht von Haller (1708-1777) über Reizphänomene (Differenzierung in Irritabilität und Sensibilität) brachten zunächst keine allgemeine Abkehr von den gewohnten, weitgehend spekulativen und der naturwissenschaftlichen Methode abgekehrten Lehren der verschiedenen medizinischen Schulen. Erst durch des Schotten John Brown wurde der Begriff der Irritabilität zum Zentralbegriff des allgemeinen Krankheitslehre erhoben. Er besagte, dass sich die lebenden Organismen von der unbelebten Materie nur durch ihre Reizbarkeit unterscheiden. Die Möglichkeit, auf äußere und innere Reize zu reagieren, hat nach Brown ihren Sitz im gesamten Nervensystem und erklärt alle physiologischen und pathologischen Reaktionen. Seine Lehren fanden im deutschen Sprachgebiet - wie an den Jenaer oder Wiener medizinischen Fakultäten - begeisterte Anhänger. Noch ohne sonderlichen Einfluss von Brown fasste 1795 der Jenaer Universitätsprofessor Christoph Wilhelm Hufeland die Vorstellungen seiner Zeit über Lebenskräfte, Reiz, Reizbarkeit und Reaktion in ein neues Konzept der Medizin zusammen, welches seine Stärke durch seine Nähe zur medizinischen Praxis besaß und viel Anerkennung fand. Seiner speziellen Krankheitslehre: der Einhaltung der Regeln für eine angemessene Lebensweise etwa durch ausreichende Bewegung oder Anwendung verschiedener Diäten wurde große Aufmerksamkeit geschenkt. Um die gleiche Zeit, in der Hufeland seine Vorstellungen von der rechten Medizin entwickelte und Brown in 
Deutschland bekannt wurde, betrat ein anderer Systemdenker mit einem neuartigen Konzept auf. Das war Samuel Hahnemann (1755-1843) mit seinem System der Homöopathie. In der Heilung wird der von natürlichen Krankheit verstimmten Lebenskraft durch Eingabe einer genau nach Symptomähnlichkeit gewählten Arzneipotenz eine etwas stärkere, ähnliche, künstliche Krankheitsaffektion beigebracht, gegen welche die instinktartige Lebenskraft eine erhöhte Energie zu richten gezwungen ist. Hahnemanns Lehre trat in Konkurrenz zu den zahllosen, grundsätzlich allopathischen (contraria contrariis curantur) Strömungen, die damals in der Medizin ihre Stimme erheben konnten, nachdem die für zwei Jahrtausende fast kanonische Humoralpathologie weitgehend ausgehöhlt schien. $\mathrm{Zu}$ dieser vielfältigen Palette der Krankheitskonzepte kam die spekulative aber in einem breiten Patientenkreis sehr populäre Lehre von Franz Anton Mesmer (1734-1815) über den tierischen Magnetismus, der in der nächsten Zeit durch einige Um- und Irrwege im 20. Jahrhundert zur Methode der planmäßigen Suggestion und Hypnose führte. ${ }^{10}$

\section{Medizinische Popularisierung}

Die zahlreichen ärztlichen Fallbeschreibungen, ärztliche und nichtärztliche Korrespondenzen, Tagebücher sowie nicht zuletzt die medizinische Aufklärungsliteratur belegen, wie groß und intensiv das damalige Interesse an Gesundheitsund Krankheitsfragen war. Im dörflichen und städtischen Miteinander redete man viel über Krankheiten. Individuelle Krankheit war viel mehr als heute ein öffentliches, allseits diskutiertes Ereignis. Unter diesen Umständen waren die medizinischen Aufklärungsschriften aus ärztlicher Feder in der breiten Bevölkerungsschicht sehr populär. Die gelehrten Ärzte übernahmen mit der Behandlung eines populären Themas die Aufgabe, die lesekundigen und nicht lesekundigen Bevölkerungsschichten anzusprechen und so zur Verbreitung der aufklärerisch-medizinischen Gedanken beizutragen. Belegt wird dies durch die ab der zweiten Hälfte des 18. Jahrhunderts stark gestiegene medizinische Buchproduktion. Das Repertoire der dazu genutzten Gattungen ist reichhaltig. Es wurden alle bekannten Medien verwendet, vom Kalender bis zu Zeitschriften, vom Buch bis zu Einblattdrucken, Flugblättern, Broschüren, Katechismen oder Lehrbüchern. Ein Teil dieser Arbeiten waren eigene Werke, andere Übersetzungen. Wichtig war dabei, die Lesenden in der Volkssprache anzusprechen. Thematisch propagiert wurden vor allem die vorbeugende Gesundheitspflege und der Kampf gegen Aberglauben. Ein wesentliches Ziel war, ein höheres Maß an Selbstbestimmung und Entscheidungskompetenz in Gesundheitsfragen zu erreichen. Die Adressaten waren ärztliche Kollegen, Heilkundige wie Hebammen oder Wundärzte, städtische und länd-

liche Unterschichten, also die Gesamtheit der Bevölkerung. ${ }^{11}$ In die medizinische 
Aufklärungsarbeit wurden auch Landpfarrer, städtische und dörfliche Priester, Schulmeister und Lehrer einbezogen. Die sog. pastoralmedizinischen Schriften aus ärztlicher Feder waren an sie adressiert. ${ }^{12}$ Pfarrer und Lehrer spielten bei der Popularisierung ab Ende des 18. Jahrhunderts gleichfalls eine wichtige Rolle, besonders in der Popularisierung der Pockenimpfung. ${ }^{13}$

Für eine neue und wichtige, eher wissenschaftliche Gattung der medizinischen Aufklärungsliteratur standen die ab Ende des 18. Jahrhunderts erscheinenden medizinischen Topographien. Sie beschrieben die sanitären Verhältnisse einer Stadt oder eines Komitats und boten gleichzeitig eine Schilderung der örtlichen geographischen, wirtschaftlichen, gesellschaftlichen und kulturellen Gegebenheiten. ${ }^{14}$

Die von den Ärzten verfassten aufklärenden Werke wurden sehr oft mit finanzieller Unterstützung eines Adeligen veröffentlicht (Antal Károlyi, György Festetics, Ferenc Széchényi). Mit Hinsicht auf die Herausgebertätigkeit ungarischsprachiger medizinischer Aufklärungsliteratur stand an erster Stelle, sogar vor Pest-Ofen, das Zentrum der Familie Károlyi in Nagykároly, welches zugleich der geistige und wirtschaftliche Hauptsitz des Komitats Szatmár war. Hier erschien 1771 das von József Csapó verfasste Werk über Kinderheilkunde, ${ }^{15} 1772$ die Übersetzung des Europaweit bekannten Buches von Simon André Tissot, ${ }^{16}$ sowie 1783 die Übersetzung des ursprünglich auf Latein geschriebenen Werkes des aus Luxemburg stammenden Varasdiner Komitatsarztes Johannes La Langue über die Heilwasser Ungarns. ${ }^{17}$

Die Stadt Debrecen war in Verbindung mit der Herausgebertätigkeit ungarischsprachiger medizinischer Literatur ein ebenso bedeutendes Zentrum. ${ }^{18}$ Nach 1790 trafen sich die Mitglieder des berühmten botanischen Kreises - Mihály Csokonai Vitéz, Mihály Fazekas, Sámuel Diószegi, Samuel Glosius, József Csapó unter der Leitung von János Földi im Hause des bekannten, England bereisten Arztes, István Weszprémi.

Die Beschäftigung mit dem Genre medizinischer Aufklärungsliteratur lässt mannigfaltige Schlüsse über die Vorstellungen der ärztlichen Verfasser über die erhofften Leser und über das Bild von der Qualität und Bedeutung medizinischer Wissenschaft und Praxis zu, das man ihnen vermitteln wollten. Solche Schriften bildeten zudem eine der beliebtesten literarischen Gattungen überhaupt. Die erfolgreicheren unter ihnen erlebten zahlreiche Auflagen und Übersetzungen, deren Autoren in ganz Europa berühmt waren. Das lässt ein breiteres Lesepublikum vermuten. Aber der bloße Blick auf die einschlägigen Texte gibt nur wenig Aufschluss über deren tatsächliche Wirkkraft und Bedeutung im Vergleich zu anderen Quellen medizinischen Laienwissens. ${ }^{19}$ Medizinisches Laienwissen wurde auf vielfältigen Wegen weitergegeben und im alltäglichen Umgang mit Krankheit immer wieder erneut angewandt und bestätigt. Medizinische Popularisierung in der spezifischen Form von populären, gesundheitsaufklärerischen Texten spielte 
dabei allem Anschein nach keine erstrangige Rolle im Vergleich zu den anderen, vorwiegend oralen und nonverbalen Vermittlungswegen. ${ }^{20}$

\section{Kazinczys Mediziner-Freundeskreis als Repräsentant der medizinischen Elite des Königreichs Ungarn}

Die Ärzte aus dem Freundeskreis des in Széphalom lebenden Literaten repräsentieren das anspruchsvolle Interesse an kulturellen Erfahrungen, das enzyklopädische Wissen, den in der Gesellschaft eingenommenen Status und den gesellschaftlichen Rang, somit all das, was für eine kleinere Elitegruppe der akademischen Ärzteschaft im Zeitalter von Kanzinczy kennzeichnend war. Es handelte sich dabei um Persönlichkeiten, die für ihre Weitsichtigkeit, ihr Studium an ausländischen Universitäten und das erlangte Wissen, ihre ausgedehnten Sprachkenntnisse, ihre beachtenswerten fachliterarischen Leistungen und für ihre aktive Präsens im öffentlichen Leben bekannt waren. Einige von ihnen waren korrespondierende Mitglieder ausländischer Gelehrtengesellschaften und galten, da sie häufig Freimaurer waren, als Mitgestalter der öffentlichen Meinung der Zeit. Unter ihnen gab es Anhänger sowie Gegner von verschiedenen medizinischen Theorien und sich manchmal gegenseitig ausschließenden therapeutischen Anweisungen, d.h. sie waren Stahlianer, Befürworter oder Leugner des Brownianismus, des tierischen Magnetismus, der Boerhaavschen Iatrichemie, der Hufelandschen Makrobiotik.

Zweifellos war József Szentgyörgyi (1755-1832) der treueste Freund von Kazinczy. Die beiden verband eine intensive Briefkorrespondenz. Ihr Briefwechsel begann 1802 durch die Vermittlung einer gemeinsamen Bekannten, der Ehefrau von Gábor Spáh. Obwohl die beiden im Zusammenhang mit Fragen der Spracherneuerung grundlegend unterschiedliche Standpunkte vertraten, verband sie eine tiefe, nahezu leidenschaftliche Freundschaft.

Szentgyörgyi besuchte zwischen 1772 und 1787 die unteren und mittleren Schulklassen in Komorn, Pressburg, Nagykőrös und Debreczin. Nach dem Abschluss der Schuljahre im Debrecziner Reformierten Kollegium, verbrachte er hier noch einige weitere Jahre als Erzieher. Später zog er nach Cegléd, wo er drei Jahre lang im Dienste der örtlichen reformierten Kirche stand. Von hier aus reiste er 1790 nach Jena, wo er 7 Semester an der medizinischen Fakultät studierte. Er besuchte unter anderen die Lehrveranstaltungen der Professoren Lenz und Hufeland. Szentgyörgyi schloss Freundschaften mit mehreren seiner ungarischen Landsleute, so auch mit dem später berühmt gewordenen Frauenarzt János Zsoldos, aber auch Schiller kannte er gut. Im Laufe seiner Studienjahre besuchte er viele deutsche Städte, unter anderen Halle, Erlangen, Leipzig, Weimar. Neben der Beherrschung der lateinischen, deutschen und französischen Sprache lernte er 
hier Italienisch und Englisch. 1794 verließ er Jena und reiste nach Wien, wo er als Praktikant im Bürgerspital und im Gebärhaus die nötigen klinischen Erfahrungen sammelte, und schließlich erhielt er den Doktortitel an der medizinischen Fakultät der Universität Wien. 1795 kehrte er nach Ungarn zurück und ließ sich in Győr nieder, wo er mit Hilfe seines Jugendfreundes, des späteren evangelischen Pastors János Kis, für das Wohlergehen eines vornehmen Klientenkreises sorgte. Er heiratete die Tochter des örtlichen Notars, Katalin Sólyom. Nach dem Tod von István Weszprémi 1799 wurde in Debreczin die Stelle des Mediziners frei. Szentgyörgyi nahm die Einladung an und versah bis zu seinem Lebensende die Aufgaben des Stadtarztes. Er erhielt eine Bezahlung in Höhe von 400 Forint, 1803 konnte er bereits einen Weingarten und ein eigenes Haus kaufen. Er war korrespondierendes Mitglied der ansehnlichen und von Goethe geleiteten Jenaer Mineralogischen Gesellschaft. ${ }^{21}$ Während der Ausübung seiner erfolgreichen medizinischen Praxis, verfasste er im Auftrag des Debrecziner Reformierten Kollegiums sein einziges Buch. Dieses reich illustrierte Werk erschien 1803 in Form eines Lehrbuches des Kollegiums, und auch Kazinczy schrieb in seinem an János Kis gerichteten Brief vom April 1803 anerkennend über das Werk. ${ }^{22}$ Auf Grund der Korrespondenz von Szentgyörgyi können wir uns ein genaues Bild über den alltäglichen Tagesablauf eines zeitgenössischen Arztes machen. Aus einem an seinen jüngeren Bruder gerichteten Brief aus dem Jahr 1800 erfahren wir, dass er sich bereits am frühen Morgen auf den Weg machte, um seine Patienten aufzusuchen, und bis zur Mittagszeit unterwegs war. Nach dem Mittagessen gönnte er sich eine kurze Pause, um dann mit seiner Arbeit fortzufahren und die medizinischen Gutachten, Berichte, Patientenbriefe zu verfassen. Nach $4 \mathrm{Uhr}$ nachmittags begann er seine Abendvisite, die oft bis $6 \mathrm{Uhr}$ andauerte, die Abendstunden widmete er anschließend seinen Studien. Auf Grund seiner ausgedehnten Korrespondenz können wir feststellen, dass er bis zu seinem Lebensende intensive Beziehungen zu seinen ungarischen und ausländischen Freunden pflegte. ${ }^{23}$

Ein weiterer treuer Freund Kazinczys aus seiner näheren Umgebung war der Kaschauer Stadtarzt, József Viczay (1746-1810). Er heilte oftmals erfolgreich die Krankheiten, an denen Kazinczy litt. Auch die aktive Freimaurertätigkeit, die sowohl für Kazinczy als auch für Viczay kennzeichnend war, vertiefte ihre Freundschaft noch mehr. Viczay war Gründungsmitglied der Intrepida Virtutis Loge, die unter der Leitung des Grafen Lajos Török 1793 in Kaschau gegründet wurde. 1794 wurde er zum stellvertretenden Großmeister gewählt. Unter den Mitgliedern der Loge finden wir Grafen, Barone (der General Baron Gábor Splényi, der kaiserlich königliche Kämmerer und späterer Obergespan des Komitats Zips, Graf Antal Csáky) sowie Lehrer, Ingenieure, Kaufleute und Handwerksmeister. ${ }^{24}$

Viczay war bis zu seinem Tod praktizierender Arzt in Kaschau und Umgebung. Es lässt sich keine wirklich nennenswerte fachliterarische Publikation mit seinem Namen verbinden. Nach 1795 trafen sich die einstigen Mitglieder der 
Loge im Gartenhaus seines Kauschauer Hauses. Es ist sehr wahrscheinlich, dass er selbst nach der Auflösung der Loge mit der Aufbewahrung deren Dokumente betraut wurde. Als Anerkennung der Verdienste von Viczay und nicht zuletzt als Ausdruck des Respekts und tiefer Freundschaft beauftragten die einstigen Logenmitglieder 1803 Sámuel Czetter damit, einen Stich über Viczay anzufertigen, den der Künstler auf Grund eines von einem unbekannten Künstler namens Szekeres gemalten Porträts auch fertig stellte. Im Juli 1803 schrieb Kazinczy in einem an Lajos Schedius gerichteten Brief wie folgt über dieses Vorhaben:

Bei meiner Durchreise in Debreczin theilte ich einen Abdruck der Viczayschen Kupferplatte auch H. Doktor Szentgyörgyi mit, und erzählte ihm zum Lobe meines Freundes unter anderen auch, dass im Senate der Stadt Kaschau durch einen Mann, den Viczay eben vom Tode gerettet, die Motion gemacht wurde, diesem würdigen Manne einen Beweis der öffentl. Dankbarkeit, auf die er so gerechte Ansprüche hat, zu geben (...) es wurde beschlossen, ihn durch einen geschickten Mahler mahlen zu lassen, und das Bild in dem Saale des Senats aufzustellen. ${ }^{25}$

Nachdem Viczay 1810 in Kaschau gestorben war, ließen seine Freimaurerbrüder eine Kapelle über seine Grabstätte errichten. Und an der Stelle, wo die brüderlichen Zusammenkünfte stattfanden, also im Garten seines Kaschauer Hauses, stellten sie seine Büstenstatue auf, deren Aufschrift von József Dessewffy auf Ungarisch, Deutsch, Latein verfasst wurde. Die Überlieferung seines Lebenslaufes verdanken wir seinem treuen Freund Kazinczy, der diesen niederschrieb. ${ }^{26}$

Eine ähnliche freundschaftliche Beziehung pflegte Kazinczy mit dem in Komorn tätigen Sámuel Nagy (?-1810). Nagy besuchte ebenfalls das Debrecziner Reformierte Kollegium. Er erhielt später in Wien sein medizinisches Diplom und machte auch hier im Bürgerspital sein Praktikum. Er verbrachte mehrere Semester an der Universität. Er wurde zuerst Sekretär, später korrespondierendes Mitglied der Jenaer Mineralogischen Gesellschaft in Jena. Er wurde ebenfalls in die „Gelehrten Gesellschaft von Komorn“ aufgenommen, einem von József Péczeli um die Zeitschrift „Mindenes Gyüjtemény“ (Sammlung von Allerlei Dingen) organisierten literarischen Kreis. Als Arzt leistete Sámuel Nagy Bedeutendes auf dem Gebiet der medizinischen Aufklärung des ,Volkes von Komorn“. Er war unter den ersten, die Menschen über die Vorteile der Pockenimpfung zu überzeugen versuchten, somit die Impfung zu popularisieren. Um mit gutem Beispiel voranzugehen und die Bedeutung sowie die Gefahrlosigkeit dieses Verfahrens zu verdeutlichen, impfte er sein eigenes Kind. Ein vorzügliches Beispiel für die Philanthropie der Zeit ist, dass er sein medizinisches Aufklärungsschrift über die Pockenimpfung auf eigene Kosten veröffentlichen lie $3 .^{27}$ Im Vorwort dieser kleinen Publikation in Heftformat sagte er eindeutig aus, dass die Pocken in nur wenigen Jahren aus Europa verbannt werden könnten, wenn die Regierung die Impfung 
obligatorisch machen würde und der ,gemeine Mann“ davon überzeugt werden könnte, das die Impfung nötig wäre. Seiner Meinung nach sollten die Gutsherren und die Geistlichen diese Aufgabe übernehmen und zwar dadurch, dass sie mit gutem Beispiel vorangingen und eine aufklärende Tätigkeit ausübten, um so die Menschen zu belehren. Sein auf Ungarisch verfasstes Werk sollte hierzu eine bedeutende Hilfe geben.

Kazinczy schreibt in seinen Briefen des Öfteren mit großer Anerkennung über József Cseh-Szombati (1748-1815). Obwohl Kazinczy und Cseh-Szombati nicht miteinander korrespondierten, verfolgte der Meister aus Széphalom aus der Ferne die erfolgreiche Heiltätigkeit des Pester Komitatsphysicus. Cseh-Szombati besuchte nach dem Abschluss seiner Schuljahre in Pressburg und Debreczin zwischen 1777 und 1782 verschiedene renommierte ausländische Universitäten, um Medizin zu studieren. Er hielt sich längere Zeit in der niederländischen Stadt Franeker auf, wo er Camper, den berühmten Gelehrten-Arzt der Zeit, kennen lernte und sich mit ihm anfreundete. Danach verbrachte er zwei Jahre in Göttingen, an der fortschrittlichsten Lehr- und Forschungsuniversität der Zeit. 1780 kehrte er wieder nach Franeker zurück, von hier aus führte ihn sein Weg über verschiedene deutsche, französische und niederländische Städte, bis er schließlich in Wien ankam. Seinem deutschsprachigen Reisebericht, der in der Manuskriptensammlung des Reformierten Kollegiums in Debrecen aufbewahrt wird, können wir entnehmen, dass seine Aufenthalte in diesen Städten in erster Linie dazu dienten, seine fachwissenschaftlichen Kenntnisse zu erweitern. Demnach suchte er Hospitale auf und sah sich die naturwissenschaftlichen Sammlungen und die botanischen Gärten an. 1782 wurde ihm der Doktortitel an der medizinischen Fakultät der Universität Wien verliehen, und noch im gleichen Jahr kehrte er nach Ungarn zurück. Er ließ sich in Pest nieder, wo er vom Jahre 1784 bis 1788 als Komitatsphysicus, dann als ein städtischer praktizierender Arzt tätig war. Cseh-Szombati setzte sich mit aller Kraft daran, im Komitat Pest die Menschen mit den Vorteilen und der Bedeutung der Pockenimpfung bekannt zu machen, genauso wie Sámuel Nagy dies in Komorn tat. Den Erfolg seiner Tätigkeit können wir daran ermessen, dass er ab 1801 jährlich 400 Personen impfte. Ab 1782 war er Mitglied in mehreren Pester und später Ofner Freimaurerlogen. In seinem Testament hinterließ er dem Debrecziner Reformierten Kollegium eine Stiftung in Höhe von 25.000 Forint, die für die Modernisierung des Unterrichts in den Fächern Chemie, Botanik und Mineralogie verwendet werden sollte. Diese Stiftung ermöglichte, dass in Debreczin ein botanischer Garten angelegt werden konnte. ${ }^{28}$

Aus dem Kreise der jüngeren Generation ist die Person von Mihály Kováts (1768-1851) hervorzuheben, der ebenfalls zum Freundeskreis von Kazinczy zählte. Mihály Kováts verfügte über das enzyklopädische Wissen seiner Zeit, beherrschte 5 Sprachen (Latein, Griechisch, Deutsch, Französisch, Englisch), und war als Gelehrter-Arzt bekannt für seine ausgedehnte fachwissenschaftliche Tä- 
tigkeit. Die in der Manuskriptensammlung der Ungarischen Akademie aufbewahrten Memoirenschriften von Kováts erlauben uns einen Einblick in seine Jugendjahre und seine Studien. Er kam 1768 in einem kleinen Dorf im Komitat Abaúj zur Welt. Er besuchte die Mittelschule im Kollegium von Sárospatak und später in Leutschau. Er lernte Latein, Griechisch, Deutsch, Französisch und war auch musikalisch talentiert - er spielte Klavier und Geige. Sein medizinisches Diplom erhielt er an der Pester Fakultät und verbrachte anschließend zwei Jahre in Wien, wo er anfangs im Bürgerspital, dann im Josephinum klinische Erfahrungen sammelte. Er war ebenfalls Praktikant - was zu der Zeit ungewöhnlich war - im Gebärhaus, im Fidelhaus und auch im Narrenturm. Er wandte sich mit großem Eifer der Aneignung von Kenntnissen im Bereich der praktischen Geburtenhilfe zu. Später besuchte er auch die deutschen Universitätsstädte (Halle, Leipzig, Göttingen, Jena usw.). In dieser Zeit lernte er Englisch. Seine praktischen Studien beendete er damit, dass er ein Semester wiederum im Josephinum verbrachte, wo er den Versuch unternahm, die zeitgenössischen Techniken der chirurgischen Eingriffe zu erlernen. Die Mitglieder der Generation, der auch Kováts angehörte, betrachteten es bereits als eine wichtige Voraussetzung, dass sie neben der Aneignung von theoretischem Wissen auch praktisch-technische Kenntnisse besitzen. 1795 kehrte er nach Ungarn zurück und war bis zu seinem Lebensende in Pest als praktizierender Arzt und als gelehrter Fachschriftsteller tätig. Er unternahm lediglich kürzere wissenschaftliche Reisen durch das zeitgenössische Ungarn. Er betrachtete - laut seiner persönlichen Bekenntnisse - das Heilen der Menschen und die Veröffentlichung und Verbreitung der Wissenschaften in ungarischer Sprache als sein Lebensziel. Als praktizierender Arzt genoss er bedeutendes Ansehen und erhielt aus allen Teilen des Landes Anfragen, in denen er gebeten wurde, die Kranken aufzusuchen. Er übersetzte 1798 das von Hufeland verfasste Werk mit dem Titel „Über die Kunst das Leben zu verlängern“ und ergänzte es mit seinen eigenen, während seiner medizinischen Tätigkeit in Ungarn angesammelten Erfahrungen, um so zur erfolgreichen Heilung der Krankheiten, die er während seiner medizinischen Tätigkeit begegnete, beizutragen. ${ }^{29}$ Dieses Werk erlebte in deutschen Ländern zahlreiche Ausgaben. Kováts übersetzte dieses Buch insgesamt dreimal, wobei er jeweils die neueste deutsche Ausgabe als Vorlage benutzte. Er galt als ein aktiver Propagator der Pockenimpfung und zog mit allen Mitteln gegen die üblen Gewohnheiten der überall im Land herumziehenden Heilkundigen aller Art. ${ }^{30}$

Neben seiner Tätigkeit auf dem Gebiet der Heilkunde wurden in erster Linie seine bedeutenden Verdienste in den Wissenschaftsbereichen Chemie und Mineralogie anerkannt. 1808 erschien sein vierbändiges Chemiewerk, das in Hinblick auf die Herausbildung der ungarischen Fachsprache der Chemie Bedeutendes leistete. ${ }^{31}$ Seine Übersetzung des Buches von Professor Gren aus Halle mit dem Titel „Grundriss der Chemie“" war kein selbstständiges Werk. Als er 1808 mit der 
Übersetzung fertig wurde, sandte er diese als erstes Kazinczy zu, damit Kazinczy den Text „mit Hinsicht auf die Übertragung in ungarische Sprache“ durchstudiert. ${ }^{32}$ Kováts war der Verfasser des ersten ungarischsprachigen Grundwerkes zur Gerichtsmedizin. Für die Zusammenstellung seines mineralogischen Lexikons $^{33}$ durfte er die Würdigung der Jenaer Mineralogischen Gesellschaft entgegennehmen.

Im Laufe seiner aufklärenden medizinischen Tätigkeit trat er entschieden gegen jede Art der pseudomedizinischen Strömungen auf. Gegen den Magnetismus von Mesmer zog er reglerecht zu Felde. ${ }^{34}$ Er wies auch die Lehren von Hahnemann zurück. 1826 verfasste er sein Werk mit dem Titel „Antiorganon“, ${ }^{35}$ welches die Gegenschrift zu dem Grundwerk der Lehren von Hahnemann, „Organon" war.

Wir müssen hier ebenfalls auf János Dercsényi, alias Johannes Weisz (17551837) verweisen, der nach seiner Heirat mit Júlia Kazinczy, die für ihre diffizile Persönlichkeit bekannt war, der Schwager von Kazinczy wurde. Die Beziehung zwischen Kazinczy und Weisz war die Jahre hindurch oft stark belastet.

Im Taufregister ist der Name Johannes Weisz aufgezeichnet. Er stammte aus einer lutherischen Pfarrerfamilie aus dem Komitat Zips und besuchte das Lyzeum in Leutschau und Pressburg. 1780 erhielt er sein medizinisches Diplom in Wien. Nach seiner Rückkehr nach Ungarn wurde er Zisper Komitatsarzt. In dieser Zeit wurde er Mitglied des von Lajos Török, später von József Viczay geleiteten „Kaschauer Loge“. 1785 heiratete er die Schwester Kazinczys. Neben seiner medizinischen Tätigkeit wandte er sich der Erforschung der Natur, der Chemie und der Mineralogie zu. In den 1790er Jahren nahm er des öfteren an den von Tamás Mauksch, dem der Botanik zugewandten lutherischen Pfarrer aus Käßmark, organisierten Wanderungen im Gebirge Tátra teil. ${ }^{36}$ Als Anerkennung seiner medizinischen Verdienste und seiner Werke zur Mineralogie und Weinbau ${ }^{37}$ wurde ihm 1793 der Adelstitel verliehen und er erhielt das im Komitat Bereg befindliche Gut namens Debreczen sowie den Titel des königlichen Rates. Wir wissen, dass er 1802 nicht mehr praktizierte und sich mit der Zeit immer mehr den wirtschaftlichen Angelegenheiten seines Unternehmens zuwandte, das ihm bedeutenden Wohlstand sicherte. Seine Beziehung zu Kazinczy wurde mit der Zeit immer problematischer: zu einem gab es eine bedeutende Diskrepanz zwischen der finanziellen Lage Kazinczys und Weisz', wobei Letzterer auf Grund seines erfolgreichen Unternehmens immer wohlhabender wurde. Zum anderen war es die schwierige menschliche Natur von Júlia Kazinczy, die das Verhältnis zwischen ihrem Ehemann und ihrem Bruder immer weiter belastete. Seine Beziehung zu Kazinczy konsolidierte sich erst nach dem Tod der Frau. ${ }^{38}$ 
Fazit

Die akademischen Ärzte unterschieden sich durch den Status als Vertreter einer akademischen Profession von den nichtakademischen Heilkundigen. Ihr Doktortitel verlieh ihnen eine mit hohem Symbolwert verbundene gesellschaftliche Position. Die Wertschätzung für die studierten Ärzte und ihre gelehrte Medizin beschränkte sich nicht auf die individuelle Suche der Patienten. Die Ärzte gewannen zunehmend auch kollektives, gesellschaftliches und politisches Gewicht. Sie hatten das Recht, die Ausübung der Medizin durch ungeprüfte Heilkundige zu untersagen. Ein Teil von ihnen bekleidete Komitats- oder Stadtarztämter und bildete die Schicht der Bürokratenärzte. Andere ließen sich als praktizierende Stadtärzte nieder oder waren als Leibärzte an gräflichen oder fürstlichen Höfen tätig. Der Komitatsphysiker hatte seinen Platz nicht im Amtsapparat des Komitats, sondern bei den conventionatus, ${ }^{39}$ die neben den gewöhnlichen, gewählten Komitatsbeamten (wie z.B. Notar) für ein Spezialgebiet verantwortlich waren. $\mathrm{Zu}$ dieser Gruppe „conventionatus“ gehörten die Komitatsärzte, Tierärzte, Landmesser. Sie mussten über ihre Ausbildung schriftliche Dokumente (Diplom oder Attest) Vorlegen und durften ihr Amt nur für eine bestimmte Zeit bekleiden - hier aber befanden sie sich in herausragender Position, da ihre Bezahlung jener des Oberstuhlrichters entsprach und der Rang auf den des Komitatsingenieurs folgte.

Die Ärzte verstanden es vorzüglich, sich systematisch und öffentlichkeitswirksam in Szene zu setzen. Schon ihr äußeres Erscheinungsbild zielte auf Selbstdarstellung ab: Vornehme Kleidung, ein gutes Pferd oder gar eine Kutsche als Transportmittel, ein üppiges Hauswesen belegten ihren wirtschaftlichen Wohlstand. Sie hatten nicht nur Einfluss auf den Ausbau des städtischen und territorialen Medizinwesens, sondern gehörten - besonders in Oberungarn - zu den prägenden Gestalten der wissenschaftlichen und bürgerlichen Öffentlichkeit. Damit waren sie Autoren und zugleich Objekte von bevölkerungspolitischen Diskursen.

\section{Anmerkunden}

In diesem Zusammenhang wird gern von Medikalisierung als einem Teil der umfassenden Modernisierung der Gesellschaft gesprochen. Der Begriff zielt zum einen darauf ab, dass allmählich immer mehr Menschen in die medizinische Versorgung einbezogen wurden. Zum anderen bezeichnet er die Professionalisierung des Heilpersonals. Zur Geschichte und Problematik des Begriffs: Franziska Loetz (1994) 'Medikalisierung in Frankreich, Großbritannien und Deutschland, 1750-1850. Ansätze, Ergebnisse und Perspektiven der Forschung' in Wolfgang U. Eckart und Robert Jütte (ed.) Das europäische Gesundheitssystem. Gemeinsamkeiten und Unterschiede in historischer Perspektive. (Stuttgart) 123-161; Jürgen Schlumbohm (1996) 'Vom Wandel der Geburtshilfe in der Neuzeit. Wie eine Frauensache zur Wissenschaft wurde', MPG (Max-Planck-Gesellschaft) Spiegel No. 5, 39-44; Michael Stolberg (1998) 'Heilkundige. Professionalisierung und Medikalisierung' in Norbert Paul und 
Thomas Schlich (ed.) Medizingeschichte. Aufgaben, Probleme, Perspektiven (Frankfurt am Main) 69-85.

2 Zur Problematik der Durchsetzung ärztlicher Deutungsmacht im Zeitalter der Aufklärung: Michael Stolberg (2004) 'Frühneuzeitliche Heilkunst und ärztliche Autorität' in Richard van Dülmen und Sina Rauschenbach (ed.) Macht des Wissens. Die Entstehung der modernen Wissensgesellschaft. (Köln, Weimar, Wien) 111-130; Bettina Wahrig (2004) 'Globale Strategien und lokale Taktiken. Ärzte zwischen Macht und Wissenschaft 1750-1850' in Richard van Dülmen und Sina Rauschenbach (ed.) Macht des Wissens. Die Entstehung der modernen Wissensgesellschaft. (Köln, Weimar, Wien) 655-680. - In Ungarn wurden in den 1970-1990er Jahren Kurzbiographien über berühmte Ärzte und lokalhistorische Werke über den Sanitätszustand der einen oder anderen Stadt beziehungsweise Siedlung verfasst. Eine umfassende Untersuchung der ungarländischen akademischen und nicht akademischen Heilkundigen sowie der Patienten des Santitätspersonals ist aber noch ein Desiderat der ungarischen sozial- sowie medizinhistorischen Forschung. Zur Problematik der Medikalisierung der Gesellschaft Ungarns: Zita Deáky (2002) 'A hivatalos és a hagyományos gyógyitás a magyar történeti forrásokban' (Budapest). Speziell zur Medikalisierung der Geburt: Lilla Krász (2003) 'A bába történeti szerepváltozása a 18. századi Magyarországon' (Budapest); Zita Deáky und Lilla Krász (2005) 'Minden dolgok kezdete. A születés kultúrtörténete Magyarországon, XVI-XX. század' (Budapest).

3 Zur Problematik des Zusammenlebens der alten medizinischen Parktiken und neuen Methoden Lilla Krász (2007) 'Von den Normen zur Praxis - von der Praxis zu den Normen. Medizinisches Wissen in Ungarn im Zeitalter der Aufklärung' in K. Lengyel Zsolt (ed.) UngarnJahrbuch 28. Zeitschrift für interdisziplinäre Hungarologie (München) 437-458.

4 Die erste staatlich erlassene allgemeine Regelung der Tätigkeit der ungarländischen Heilkundigen wurde von der Ungarischen Statthalterei 1745 herausgegeben. Der Text des Erlasses wurde vom Pressburger Stadtarzt Johann Torkos Justus formuliert. Diese „Taxa Pharmaneutica Posoniensis" regelte die Preise der verschiedenen medizinischen Dienstleistungen sowie die Pflichten der Heilkundigen Franciscus Xaverio Linzbauer (1852-1861) 'Codex Sanitario-Medicinalis Hungariae’ (Buda) Vol. II. 214-220. Die nächste Regelung - „Planum regulationis in re Sanitatis“ - erließ 1755 ebenfalls die Ungarische Statthalterei. Mit ihr wurde für die Behörden der königlichen Freistädte und Komitate die Pflicht der Zusammenschreibung der offiziell oder inoffiziell tätigen Heilkundigen angeordnet. Den Wanderpfuschern sowie den inoffiziellen, also ungeprüften Spezialisten wurde die Durchführung von inneren Behandlungen untersagt. Die Stadt- und Komitatsverwaltungen mussten jede Operation bei der Sanitätskommission der Ungarischen Statthalterei anmelden: Franciscus Xaverio Linzbauer (1852-1861) 'Codex Sanitario-Medicinalis Hungariae' (Buda) Vol. II. 214-220. Vol. II. 303. 1770 wurde die Sanitätsnorm erlassen, die für das ganze Habsburgerreich eine einheitliche Regelung in Bezug auf die Prüfungsverpflichtungen jedes heilkundigen Berufes verfügte. Die Verordnung kriminalisierte die Anstellung nicht geprüfter oder nicht unterrichteter Heilkundiger und diskriminierte zugleich die empirischen Heilpersonen als illegale „Kurpfuscher“. Ihr ursprünglich lateinischsprachiger Text wurde in die Sprache aller ungarländischen Nationalitäten übersetzt, also ins Ungarische, Deutsche, Slowakische, Kroatische, Serbische, Russische: Franciscus Xaverio Linzbauer (1852-1861) 'Codex Sanitario-Medicinalis Hungariae' (Buda) Vol. II. 535-571.

5 Die Olitätenhändler wurden in den Quellen bereits im 16. Jahrhundert erwähnt, ihre Bedeutsamkeit zeigte sich aber noch später in ihren Wanderungen im 17. und 18. Jahrhundert. Sie reisten in bunten Husarenuniformen durch ganz Europa, von Schweden bis zum Osmanischen Reich, von der atlantischen Küste bis zum Ural. Gyula Magyary-Kossa (1929-1940) 'Magyar orvosi emlékek' (Budapest) Vol. I. 77., Vol. II. 162-167. 
6 Bettina Wahrig (2004) 'Globale Strategien und lokale Taktiken. Ärzte zwischen Macht und Wissenschaft 1750-1850' in Richard van Dülmen und Sina Rauschenbach (ed.) Macht des Wissens. Die Entstehung der modernen Wissensgesellschaft. (Köln, Weimar, Wien) 670-672.

7 Von den Verfassern der vitalistischen Leitkonzepten wurde der Philosoph der Frühaufklärung Christian Wolff (1679-1754) aufgenommen und zitiert. Seine Schriften tragen vielfach den charakteristischen Titel „Vernünftlige Gedanken von ...“: Vernünftige Gedanken von den Kräften des menschlichen Verstandes und ihrem richtigen Gebrauche in der Erkenntnis der Wahrheit. Halle, 1712. Vernünftige Gedanken von Gott, der Welt und der Seele des Menschen, auch allen Dingen überhaupt. Frankfurt und Leipzig, 1719. Vernünftige Gedanken von der menschen Thun und Lassen, zur Befärderung der Glückseligkeit. Halle, 1720. Vernünftige Gedanken von dem gesellschaftlichen Leben der Menschen, und insonderheit dem gemeinen Wesen. Halle, 1721. Vernünftige Gedanken von den Absichten der natürlichen Dinge. Frankfurt, 1723. Vernünftige Gedanken von den Wirkungen der Natur. Halle, 1723.

8 Karl Ed. Rotschuh (1978) 'Konzepte der Medizin in Vergangenheit und Gegenwart' (Stuttgart) 240.

9 Von den Arzneien haben sich bis heute nur die Hoffmannstropfen erhalten, ein gemisch von 1 teil Aether mit 3 teilen Alkohol. Klaus Pfeifer (2000) 'Medizin der Goethezeit. Christoph Wilhelm Hufeland und die Heilkunst des 18. Jahrhunderts' (Köln, Wiemar, Wien) 3-5.

10 Nach der Theorie von Mesmer beeinflussen die gegenseitigen Anziehungskräfte der Planeten das menschliche Nervensystem. Magnete sollten durch ihre natürlichen Strahlen ebenfalls eine heilsame Wirkung auf tierische und menschliche Organismen haben. Diese These legte er in seinem „Sendschreiben an einen auswärtigen Arzt über die Magnetkur“ (Wien, 1775) nieder. Karl Ed. Rotschuh (1978) 'Konzepte der Medizin in Vergangenheit und Gegenwart' (Stuttgart) 318, 483.

11 Repräsentativ sind in Ungarn für die an die Laien beziehungsweise den gemeinen Mann adressierte medizinische Aufklärungsliteratur die Werke des schon erwähnten Nógráder Komitatsphysikers János Dániel Perliczy (1740) Medicina pauperum (Buda) und des Hofarztes des Grafen Ferenc Széchényi, József Kiss (1794) 'Egésséget tárgyazó Katechismus' (Sopron). Eine systematisch-enzyklopädische Darstellung der zeitgenössischen naturwissenschaftlichen Kenntnisse ist István Mátyus (1787-1793) 'Ó és Új Diaetetica' (Pozsony). Vol. I-VI.

12 Der erfolgreichste Arzt im Bereich der Pastoralmedizin war der Physiker des Komitats Komorn (Komárom), Sámuel Zay (1810) 'Falusi orvos pap' (Pozsony).

13 Das Thema der Pockenimpfung behandelte der Komorner Stadtarzt Sámuel Nagy (1801) 'Az oltalmazó himlőről' (Komárom). Selbst Pfarrer schrieben illustrierte „Noth- und Hilfsbüchlein“, so der Landpfarrer im ostungarischen Debrecen, dann in Munkács, János Kömlei (1790) 'Szükségben segitö könyvetske' (Pest).

14 Die frühesten medizinischen Topographien entstanden in Oberungarn. Die erste Topographie in deutscher Sprache stammt vom Preßburger Stadtarzt Johannes Torkos Justus (1764) 'Bericht von der königlichen des Königreichs Hungarn Freys-Stadt Preßburg. Lage, Wässern und Lufft' (Preßburg); Johann Georg Hoffinger (1791) 'Vermischte medicinische Schriften. Medicinische Topographie der königl. Freien Bergstadt Schemnitz in Niederhungarn' (Wien); Sámuel Benkő (1794) 'Medizinische Ephemeriden von den Jahren 1780-1790. Aus dem Lateinischen übersetzt und mit Topgraphie Miskoltz vermehrt' (Wien).

15 József Csapó (1771) 'Kisgyermekek isputálja' (Nagykároly).

16 Die ungarische Übersetzung von Tissot: Márton Marikowski (1772) 'A néphez való tudósitás, miképen kelljen a maga egészségére vigyázni' (Nagykároly). Das Originalwerk: Samuel August Tissot (1761) 'Avis au peuple sur la santé' (Lausanne).

17 János La Langue (1783) 'A magyarországi orvos vizekröl, és a betegségekben azokkal való élésnek szabott módjairól a szegények kedvekért' (Nagykároly). 
18 In Debreczin erschien zum Beispiel István Weszprémi (1766) 'Bába mesterségre tanitó könyv' (Debrecen).

19 Zum Programm einer komparativen historischen Analyse von Popularisationsprozessen aus der Laienperspesktive vgl. Michael Stolberg (2003) 'Homo patiens. Krakheits- und Körpererfahrung in der frühen Neuzeit' (Köln, Weimar, Wien) 108-112.

20 In unserer Forschung eröffnen zeitgenössische Selbstzeugnisse und allem voran die Patientenbriefe einen wertvollen Zugang von Wissensquellen jener gebildeten und wohlhabenderen Schichten, auf die die medizinische Aufklärungsschriften ohnehin in erster Linie abzielten.

21 Aus den Jahrbüchern der Gesellschaft erfahren wir, dass in 1801 von den 595 registrierten Mitgliedern 73 ungarischer Abstammung waren, und unter diesen befanden sich zahlreiche Ärzte. Klára Benedek (1942) 'A jénai Ásványtani Társaság magyar tagjai. Levelek a magyar felújulás szellemi életének történetéhez' (Budapest); Dezső Gurka (2009) 'Az ásványtani tájékozódás szerepe a jénai konstellációban' in Dezső Gurka (ed.) A romantika terei. Az irodalom, a müvészetek és a tudományok intézményei a romantika korában (Budapest) 107-118.

22 János Váczy (ed.) (1890-1911) 'Kazinczy Ferenc levelezése' (Budapest) Vol. III. 456.

23 Márta Halmy (1991) 'Szentgyörgyi József orvosi levelei Kazinczy Ferenchez' in A debreceni Déri Múzeum évkönyve, No. 69, 335-336. Weiteres zu seiner Person: 'Kazinczy Ferenc levelei Szentgyörgyi Józsefhez' (1857) József Najza (ed.) (Pest); György Diósadi Elekes 'Szentgyörgyi József debreceni orvos (1765-1832) élete és orvosi levelei' in Orvostörténelem, 1937. 95-96, 119-120, 141-144, 166-168, 190-192, 215-216, 239, 262-263, 287.

24 Lajos Abafi (1900) 'A szabadkömüvesség története Magyarországon' (Budapest) 343-349.

25 János Váczy (ed.) (1890-1911) 'Kazinczy Ferenc levelezése' (Budapest) Vol. III. 478.

26 Lajos Abafi (1900) 'A szabadkömüvesség története Magyarországon' (Budapest) 349.

27 Sámuel Nagy (1801) 'Az oltalmazó himlöröl' (Komárom).

28 Reisejournal von József Cseh-Szombati in Manuskript unter dem Titel Bemerkungen über meine Reise von Wien aus, vom 26-ten Dezember 1790'. Archiv des Debrecziner Reformierten Kollegiums, Cs. 3472. Gábor Báthori (1821) 'Emlékeket kövekkel megrakott temetö kert: vagy olly halotti prédikatzok melleket külömb 'külömb' helyeken s' idöben elmondott' (Pest) 35-69; Abafi Lajos (1900) 'A szabadkömüvesség története Magyarországon' (Budapest) 190, 214, 275, 281, 286, 295, 339, 364, 366, 367, 388, 389, 396, 404, 408.

29 Das Werk von Hufeland wurde mit finanzieller Unterstützung des Grafen Georg Festetics herausgegeben. Die allererste Ausgabe: Mihály Kováts (1798) 'Az ember élete meg-hoszszabbitásának mestersége' (Pest).

30 Zur Person von Mihály Kováts seine eigenhändige kurze Biographie in der Handschriftensammlung der Ungarischen Akademie der Wissenschaften, Briefwechsel, 85/1833.

31 Mihály Kováts (1808) 'Chémia vagy a természet titka' (Pest).

32 János Váczy (ed.) (1890-1911) 'Kazinczy Ferenc levelezése' (Budapest) Vol. V. 332.

33 Mihály Kováts (1822) 'Lexikon mineralogicum' (Pest).

34 Mihály Kováts (1802) 'A mágnesesség méröserpenytyüje' (Pest).

35 Mihály Kováts (1830) 'Antiorganon' (Pest).

36 Tagebuch von Thomas Mauksch. Handschriftensammlung des Evangelischen Lyceums in Käsmark, MS 1773 MS-25.

37 Johann Dercseny von Derzen (1796) 'Ueber Tokay's' Weinbau! (Wien).

38 Die neueste biographische Arbeit zur Person János Dercsényi: László Kiss (2009) 'Kazinczy sógora, Zemplén föorvosa: Dercsényi János' (Budapest, Debrecen).

39 Conventionatus ist ein zeitgenössischer Begriff für die befristeten fachgebildeten Amtsträger. 